\title{
Amoebic keratitis: a clinicopathological case report
}

\author{
O.-E. LUND, F. H. STEFANI, AND W. DECHANT \\ From the University Eye Hospital, University of Munich
}

SUMMARY A 22-year-old farmer with recurrent corneal erosions and keratoiritis underwent a penetrating keratoplasty. Investigations carried out on the corneal disc revealed an amoebic keratitis, which is considered to be a very rare condition.

An unusual case of corneal ulcer with hypopyon and subsequent corneal perforation was demonstrated by A. Hamburg in 1972 (unpublished communication). At that time we were not aware of the amoebic problem and thus classified the cystic bodies in the inflamed anterior parts of the eye as 'unknown' organisms. The presentation by Professor Norman Ashton during the 14th Annual Meeting of the European Ophthalmic Pathology Society in Oslo in 1975, as well as the superb papers presented at the symposium on Infections of the Eye by Acanthamoeba during the 60th Oxford Ophthalmological Congress in July 1975 (Jones et al., 1975a, b; Nagington, 1975; Watson, 1975), made us familiar with this eye infection. Infections due to the widely distributed free-living acanthamoebae have only rarely been reported, which is perhaps to be expected since they are only occasionally pathogenic.

Until now there have been no accounts of eye infections or meningoencephalitis due to acanthamoeba in Germany (Piekarski, personal communication, 1977).

\section{Case report}

While cutting a tree with a motor saw a healthy 22-year-old farmer got sawdust in his left eye, causing a scratching sensation. The next day he noticed a mild conjunctivitis with some pain, and the following day he visited an ophthalmologist, who removed a foreign body from beneath the upper tarsus. A week later the patient presented again with a keratoiritis, for which he was referred to the University Eye Hospital.

Slit-lamp examination revealed a left marginal corneal erosion in the lower temporal quadrant, a diffuse circular marginal opacification of the corneal stroma, massive hyperaemia of the conjunctiva, and

Address for reprints: Professor Dr med. O.-E. Lund, Augenklinik der Universität, Mathildenstrasse 8, D-8000 München 2, West Germany some slight swelling of the upper and lower lids. In the anterior chamber there was a positive flare with some inflammatory cells. The vitreous body and the fundus were normal. Visual acuity was found to be $5 / 10$ in this eye. $X$-rays failed to show any foreign body. Cultures and a conjunctival scraping were negative for bacteria and fungi. Local antibiotic treatment together with scopolamine hydrobromide was started, but on the next day the condition had deteriorated in that folds of Descemet's membrane were present and the visual acuity had fallen to 5/40. Fig. 1 shows the situation at that time, when coarse opaque streaks with more translucent small intervals were present in the temporal cornea. The therapeutic regimen was continued, and the changes slowly decreased within 1 week; visual acuity increased to $5 / 10$. But then keratic precipitates and an increased number of anterior chamber cells suddenly appeared, so that the patient needed to be admitted to hospital, where an increase in corticosteroid therapy led to improvement within 12 days.

Six weeks after leaving hospital the patient had to be re-admitted because the corneal condition had worsened markedly after he had received treatment for maxillary sinusitis by drainage and oral penicillin. During this time the eye treatment consisted of local chloramphenicol and scopolamine hydrobromide. Visual acuity had fallen to counting of fingers at 0.5 metre. The cornea was diffusely infiltrated with stromal thickening, folds of Descemet's membrane, and keratic precipitates. There was a marked flare in the anterior chamber and cells were ++ . Local antibiotic treatment with mydriatics was started once more, and again the condition became worse on the first day of therapy, when a round central erosion and a semilunar erosion in the lower half of the cornea developed. Within 12 days the epithelial defect closed and local corticosteroids were given; visual acuity again recovered to $5 / 40$. But after 1 week a semilunar erosion developed in the upper half of the cornea, which was resistant to therapy, 


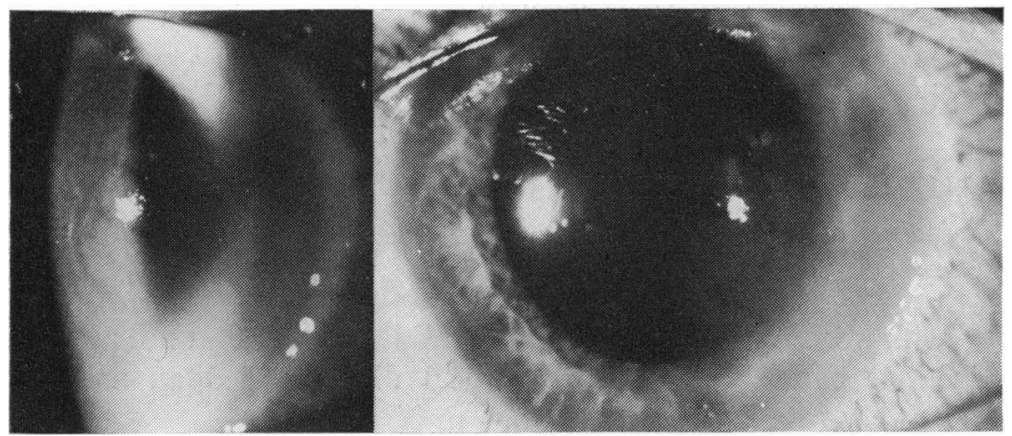

Fig. 1 Coarse radial streaks of opacities with small, more translucent intervals in the temporal half of the left cornea 10 days after trauma

and merged with a recurrence of a similar erosion in the lower half of the cornea to give a sharply outlined large central epithelial defect. At this point an uncomplicated $7-\mathrm{mm}$ penetrating keratoplasty was performed because of suspected mycotic keratitis. The postoperative course was uneventful.

Now $1 \frac{1}{2}$ years later the graft is still clear and the inflammatory reaction has not recurred.

\section{Pathological findings}

(Specimen M 609/76)

\section{LIGHT MICROSCOPY}

The formalin-fixed, paraffin-embedded corneal disc showed irregularities of the epithelial layers with some oedema and increased numbers of mononuclear round cells. Bowman's membrane was found to be defective in 2 areas where the surrounding stroma showed a marked fibroblastic reaction. In some places the reaction was reminiscent of a foreign body granuloma but without any evidence of a foreign body. The corneal stroma was moderately infiltrated by polymorphous leucocytes. Within the superficial layers of the stroma (that is, the anterior quarter) and adjacent to the defects in Bowman's membrane large clear cystic bodies were observed, some of which contained a dumb-bell-like structure. PAS stains and Grocott-Gomori silver impregnation disclosed a well-stained membrane round the cystic bodies (Fig. 2). Adjacent to these bodies there was practically no inflammatory or fibroblastic reaction. Typical trophozoites were not observed.

ELECTRON MICROSCOPY

Formalin-fixed wet material was processed for electron microscopy, which revealed free cysts in the corneal stroma with a thick outer wall and electron-dense cytoplasmic contents (Fig. 3). Other cysts were phagocytosed and broken up by leucocytes (Fig. 4); it also appeared that local fibroblasts were sharing in the phagocytic reaction.
IMMUNOFLUORESCENT TECHNIQUE

This investigation was carried out by Air ViceMarshal W. P. Stamm (Amoebiasis Diagnostic and Research Unit, St. Giles's Hospital, London) by kind arrangement of Professor Norman Ashton. The cysts showed positive fluorescence with antiserum to Acanthamoeba castellanii.

\section{Discussion}

The mechanism of amoebic infection in this case is uncertain. Most probably it was a secondary infection after a foreign body (sawdust) had damaged the cornea. The patient's work as a farmer must also be regarded as a risk factor. Although no

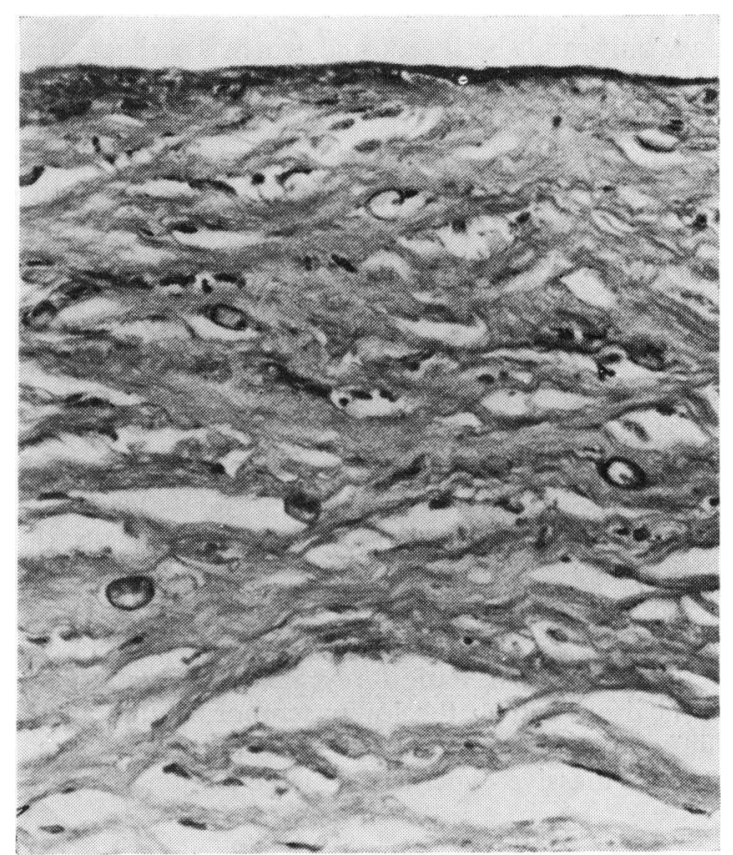

Fig. 2 Cystic organisms with thick membranes in the anterior corneal stroma. PAS, $\times 350$ 
Fig. 3 Electron micrograph of cyst lying free within the corneal stroma. $\times 5000$
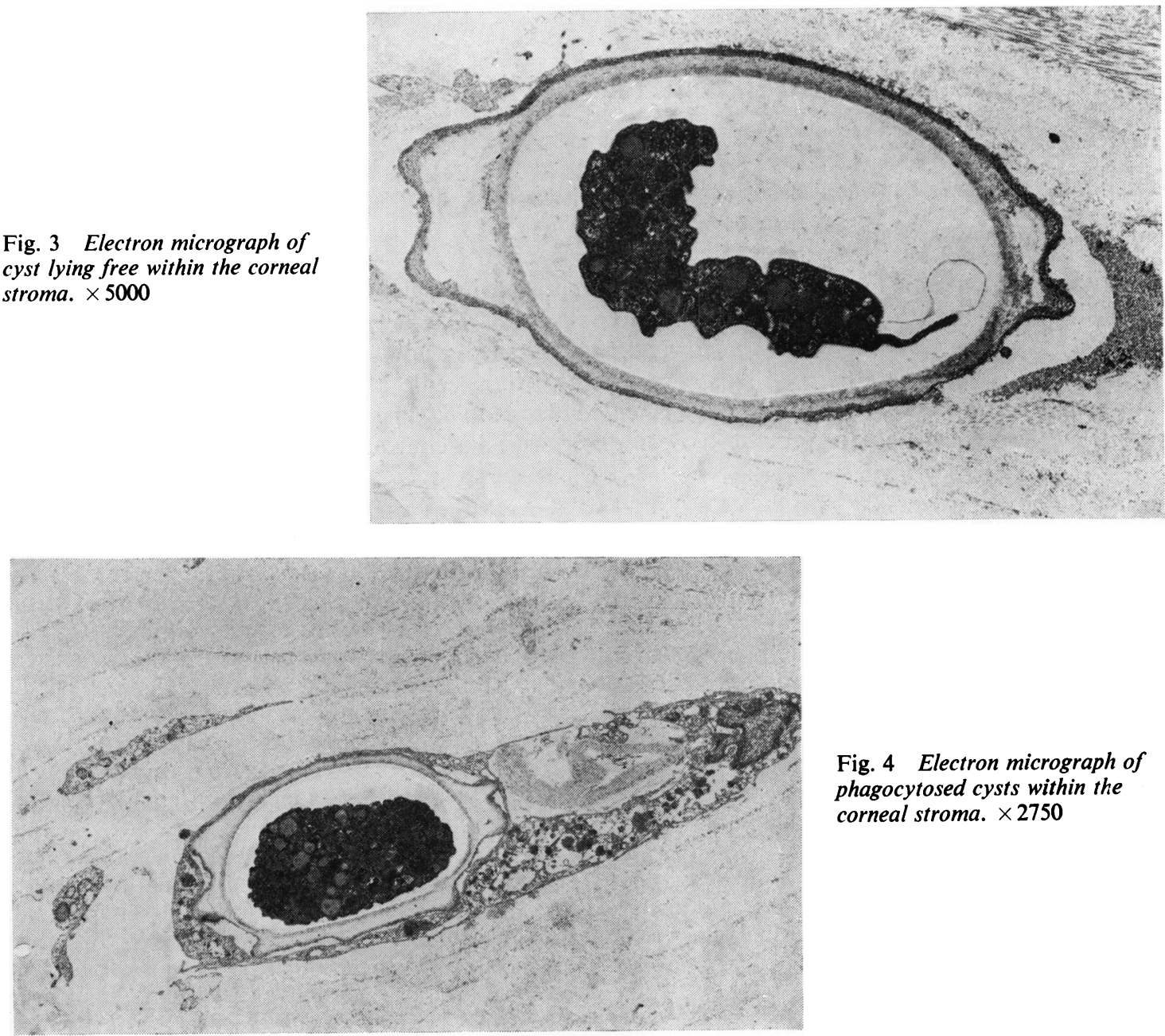

Fig. 4 Electron micrograph of phagocytosed cysts within the corneal stroma. $\times 2750$

specific treatment was given, the disease was rather slowly progressive, with recurrent erosions and moderate stromal keratitis and iritis. Along with the morphological observations we interpret this as a normal defence mechanism.

As to the frequency of amoebic infections of the eye, our experience underlines the suggestion of Ashton and Stamm (1975) that the condition is extremely rare. In a study of cases seen since late 1972 we examined 34 eyes enucleated because of endophthalmitis and 138 corneal discs with keratitis; 4 of the eyes had developed massive endophthalmitis after unsuccessful treatment for corneal ulcer. We are convinced that cysts were not overlooked, but trophozoites may have been missed.

We are most grateful to Air Vice-Marshal W. P. Stamm for carrying out the immunofluorescent test.

\section{References}

Ashton, N., and Stamm, W. (1975). Amoebic infection of the eye. A pathological report. Transactions of the Ophthalmological Societies of the United Kingdom, 95, 214-220.

Jones, B. R., McGill, J. I., and McG. Steele, A. D. (1975a). Recurrent suppurative kerato-uveitis with loss of eye due to infection by Acanthamoeba castellanii. Transactions of the Ophthalmological Societies of the United Kingdom, 95, 210-213.

Jones, D. B., Visvesvara, G. S., and Robinson, N. M. (1975b). Acanthamoeba polyphaga keratitis and Acanthamoeba uveitis associated with fatal meningoencephalitis. Transactions of the Ophthalmological Societies of the United Kingdom, 95, 221-231.

Nagington, J. (1975). Isolation of amoebae from eye infections in England. Transactions of the Ophthalmological Societies of the United Kingdom, 95, 207-209.

Watson, P. G. (1975). Amoebic infection of the eye. Transactions of the Ophthalmological Societies of the United Kingdom, 95, 204-206. 